\title{
The effect of autumn and spring planting time on seed yield and protein content of chickpea genotypes
}

\author{
Şemun Tayyar ${ }^{1 *}$, Cem Ö. Egesel ${ }^{2}$, Kemal M. Gül $^{2}$ and Hakan Turhan ${ }^{2}$ \\ ${ }^{1}$ Biga Vocational College, Çanakkale Onsekiz Mart University, 17200 Biga-Çanakkale/Turkey. \\ ${ }^{2}$ Field Crops Department, Çanakkale Onsekiz Mart University, 17020 Çanakkale/Turkey.
}

Accepted 21 April, 2008

\begin{abstract}
The objective of this study was to investigate the effects of autumn and spring plantings on seed yield and quality of chickpea genotypes. Fourteen chickpea genotypes were grown over the consecutive two growing seasons in northwest Turkey. The results showed that planting time had significant effects on the investigated traits $(P<0.05)$. Significant differences for yield were observed between autumn $(2050$ $\left.\mathrm{kg} \mathrm{ha}^{-1}\right)$ and spring (1588 $\mathrm{kg} \mathrm{ha}^{-1}$ ) plantings. Line $99-59 \mathrm{C}$ was the highest yielding genotype both in autumn $\left(2662 \mathrm{~kg} \mathrm{ha}^{-1}\right)$ and spring $\left(2000 \mathrm{~kg} \mathrm{ha}^{-1}\right)$ plantings. Seed analysis revealed that crude protein content in spring planting $(23.2 \%)$ was higher than in autumn planting $(20.5 \%)$. The highest protein content $(21.1 \%)$ was produced by genotype P-2 in autumn planting whereas line $97-73 \mathrm{C}$ had the highest content $(24.6 \%)$ in spring planting. In addition, yield was highly and positively correlated with $\mathrm{C} / \mathrm{N}$ ratio $\left(r=0.20^{\star \star}\right)$ whereas it was negatively correlated with protein $\left(r=-0.19^{\star \star}\right)$. As a result, planting time influenced yield, yield components and chemical composition of the genotypes. Autumn planting had advantages for higher seed yield and consequently higher amount of protein per harvested area.
\end{abstract}

Key words: Edible legumes, adaptation, seed quality, yield components.

\section{INTRODUCTION}

Legume seeds are the most important plant-based protein sources for human consumption, as well as for feeding animal in many developing countries. They enhance the protein content of many diets and are the major contributors of the nutritional status of the cerealbased diets (lqbal et al., 2006). In contrast to cereals, they contain adequate amounts of lysine, an essential amino acid (Farzana and Khalil, 1999; Amjad et al., 2003). Furthermore, it is of great importance in dry agricultural systems as a substitute in cereal rotations with the sustainability of annual production and in the fixation of atmospheric nitrogen by means of symbiotic nodules in their roots (Akçin, 1988; Singh, 1997).

Chickpea (Cicer arietinum L.) is one of the important edible legumes in the world, widely grown in various environmental conditions (Singh, 1997). Its seeds contain 38 - 59\% carbohydrate, $4.8-5.9 \%$ oil, $3 \%$ ash, $3 \%$ fiber,

${ }^{*}$ Corresponding author. E-mail: stayyar@comu.edu.tr. Tel: + (90) 286 3162878. Fax: + (90) 2863163733.
$0.2 \%$ calcium, and $0.3 \%$ phosphorus (Hulse, 1991). The crude protein content of chickpea seed shows a great variation, ranging between 12.6 and $30.5 \%$ (El Hardallon and Salih, 1981), depending upon genotype and/or different environmental factors.

Chickpea is traditionally planted in spring and grown without irrigation in Mediterranean countries including Turkey. However, this results in low and inconsistent yields and quality in most years. Old varieties were susceptible not only to low temperatures (both freezing and chilling) but also diseases such as Ascochyta blight and Sclerotinia sp. In order to increase yield and quality in chickpea production in arid and semi-arid regions, such as Mediterranean zone, the planting time could be shifted from spring to autumn to enable better use of soil moisture when irrigation is not available. Thus, some new chickpea cultivars resistant to frost and Ascochyta blight have been developed for these areas (Hawtin and Singh, 1984; Singh, 1997).

Various studies conducted in many parts of the world have resulted in significantly high yield in autumn plantings compared to spring plantings. According to several 
reports, these yield increases, depending upon environmental conditions ranged between 23 - 188\% (Hawtin and Singh, 1984; Calcagno et al., 1987; Singh et al., 1997; Iliadis, 2001). In other investigations carried out in Turkey, the results were in line with the above observations (Özdemir and Karadavut, 2003; Gül et al., 2005; Tayyar et al., 2005).

There have been some studies on the nutritional composition and quality of chickpea (Fernando Flores and Hernandez, 1987; El-Adawy, 2002; lqbal et al., 2006; Almeida Costa et al., 2006; Gül et al., 2008). These studies showed that chickpea is a good source of protein and carbohydrate which varies widely depending on genotype and growing conditions as well as cultural practices. The correlation between the seed yield and the seed protein content has been found to be negative for most of the legume species (Monti and Grillo, 1983). In addition, significant genotype $x$ environment interactions was observed in seed yield and some seed quality traits in chickpea (Ghirardi et al., 1974; Berger et al., 2006). Similarly, in a study with chickpea, significant $G \times E$ interactions were found for most of the quantitative characters (Gül et al., 2008).

Although there is a great deal of studies on the effects of planting time on yield and yield related traits of chickpea, their effects on contents of protein and $\mathrm{C} / \mathrm{N}$ have not yet been fully studied. Therefore, the objectives of this study were i) to investigate the potential advantages of spring and autumn planting times in chickpea, ii) to determine some seed quality traits, namely $\mathrm{C}$, and protein contents, and iii) to detect relationships among investigated components.

\section{MATERIALS AND METHODS}

Fourteen chickpea genotypes were used in the study. The set of genotypes consisted of ten breeding lines (99-59C, $98-103 \mathrm{C}, 98$ - 32C, 97 - 223C, 07 - 102C, 97 - 101C, 97 - 91C, 97 - 75C, 97 $73 \mathrm{C}$ and $97-50 \mathrm{C}$ ) obtained from ICARDA, three local populations (P-1, P-2 and P-3) and one standard variety (Cevdet bey), commonly grown in the province. Cevdet bey was registered by Aegean Agricultural Research Institute, in Izmir-Turkey. The genotypes were planted in a completely randomized block design with three replications at Dardanos Experimental Station of The Agricultural Faculty of the University of Çanakkale Onsekiz Mart, in Turkey, in two consecutive growing seasons (2004- 2005 and 2005 - 2006). The experimental soil was a sand loam and not fertilized. Some meteorological data for the experimental site were presented in Table 1. Each genotype was planted in $5 \mathrm{~m}^{2}$ plots consisting of four rows with $25 \mathrm{~cm}$ row spacing at the end of November for the autumn planting and at the beginning of April for the spring planting in both years. No inoculation with Rhizobium bacteria was done, because the field has a history of chickpea and the bacteria population was considered to be sufficient. Weeds were controlled by hand and no chemical was used to control pests. The plants were harvested and threshed in June for the autumn planting and in July for the spring planting, when the seeds were dry. To avoid edge effects 2 central rows were harvested. Plant height $(\mathrm{PH})(\mathrm{cm})$, first pod height $(\mathrm{FPH})(\mathrm{cm})$, branches/plant (BN) (numbers) and seeds/plant (PN) (numbers) were recorded from 10 plants in each plot. In addition, 1000 seed weight (1000 SW) (g) and seed yield
(Y) $\left(\mathrm{kg} \mathrm{ha}^{-1}\right.$ ) were recorded. For laboratory analyses randomly taken samples obtained from each plot were used in 3 replicates, and the seeds were ground in a Retsch-ZM 200 mill $(0.20 \mathrm{~mm})$. Crude protein and $\mathrm{C} / \mathrm{N}$ ratio in seeds were determined using a $\mathrm{C} / \mathrm{N}$ analyzer (vario EL Elementar, Hanau Germany) by DUMAS method at Field Crops Department Agriculture Faculty Georg August University in Germany. After obtaining total carbon and $\mathrm{N}$ values, $\mathrm{C} / \mathrm{N}$ ratio was evaluated and finally total protein content was calculated by multiplying $\mathrm{N}$ by 6.25 (Total protein $=\mathrm{N} \times 6.25)$.

Statistical analyses were done using SAS package program (SAS, 1999). Means were separated by LSD test.

\section{RESULTS AND DISCUSSION}

Variance analysis of the 2-year data showed significant genotype $x$ year interaction for yield and $1000 \mathrm{SW}$. Genotype $x$ planting time (PT) interaction was significant on yield, $\mathrm{C}$ and the related parameters $(\mathrm{C} / \mathrm{N}$ ratio and PO). Planting time affected all of the measured characters. Genotype term was also significant for all characters except BN (Table 2).

Comparison of seed yields by the planting time indicated a clear advantage of autumn plantings. Based on 2 year data autumn planted chickpea resulted in $29 \%$ higher seed yield than the spring planted crop (Table 3). These results are in agreement with previous findings by several researchers (Calcagno et al., 1987; Singh et al., 1997; lliadis, 2001; Özdemir and Karadavut, 2003). This higher yield could be attributed to moisture availability in the soil during pod formation and maturation period for the autumn planted crop. The plants planted in the autumn face drought stress less than those plants grown in spring. In addition to seed yield, some seed components were also significantly affected by planting time. Table 3 indicates that the average protein concentration of the genotypes at autumn planting was significantly lower $(20.5 \%)$ than those planted in spring (23.2\%). Effect of spring or autumn planting on protein content is well known in wheat. The longer starch accumulation period in winter wheat results in a higher starch concentration, consequently lower protein concentration (Kün, 1988). The reason of higher protein concentration in spring plantings could be also explained with shorter growth period during pod filling in which starch accumulation is lower compared to longer growth periods.

The response of genotypes to planting time in terms of growing seasons revealed that most of morphological parameters measured were significantly affected by year (Table 4). For instance, in autumn planting, the seed yield for the first year (3039 kg ha ${ }^{-1}$ ) was very high compared to the second year $\left(1062 \mathrm{~kg} \mathrm{ha}^{-1}\right)$. Although temperature did not vary much between the first and second years, field surface was covered by snow in the first year which reduced the detrimental effect of low temperatures on plant growth and survival. In addition, according to visual observation, occurrence of Ascochyta blight disease in the second year was also higher than the first year. Unlike the morphological characters, protein and carbon 
Table 1. Some meteorological data for the experimental area.

\begin{tabular}{|c|c|c|c|c|c|c|c|c|c|c|c|c|c|}
\hline \multirow[b]{2}{*}{ Temperature } & \multirow[b]{2}{*}{ Year } & \multicolumn{12}{|c|}{ Month } \\
\hline & & 1 & 2 & 3 & 4 & 5 & 6 & 7 & 8 & 9 & 10 & 11 & 12 \\
\hline \multirow{3}{*}{ Average maximum temp. $\left({ }^{\circ} \mathrm{C}\right)$} & 2005 & 10.0 & 8.4 & 12.6 & 17.2 & 22.7 & 27.1 & 30.4 & 30.7 & 26.8 & 19.5 & 13.9 & 12.1 \\
\hline & 2006 & 6.3 & 9.0 & 12.8 & 18.5 & 22.8 & 27.3 & 30.1 & 31.9 & 26.3 & 19.8 & 14.5 & 11.4 \\
\hline & Long & 9.6 & 9.8 & 12.3 & 17.0 & 22.3 & 27.6 & 30.4 & 30.2 & 26.1 & 20.6 & 15.2 & 11.2 \\
\hline \multirow{3}{*}{ Average temperature $\left({ }^{\circ} \mathrm{C}\right)$} & 2005 & 6.8 & 6.0 & 8.2 & 12.8 & 17.9 & 21.9 & 25.5 & 25.7 & 21.7 & 14.9 & 10.5 & 9.1 \\
\hline & 2006 & 3.1 & 5.6 & 8.7 & 13.2 & 17.7 & 22.2 & 24.8 & 26.4 & 21.3 & 16.2 & 10.4 & 7.5 \\
\hline & Long & 6.3 & 6.3 & 8.3 & 12.5 & 17.4 & 22.3 & 25.0 & 24.7 & 20.8 & 16.0 & 11.4 & 8.1 \\
\hline \multirow{3}{*}{ Average minimum temp. $\left({ }^{\circ} \mathrm{C}\right)$} & 2005 & 4.0 & 3.7 & 4.5 & 9.2 & 14.0 & 16.6 & 20.5 & 21.0 & 16.9 & 11.4 & 7.4 & 6.4 \\
\hline & 2006 & 0.3 & 2.3 & 5.3 & 9.2 & 12.7 & 16.8 & 20.1 & 21.4 & 17.1 & 13.4 & 6.9 & 4.4 \\
\hline & Long & 3.2 & 3.2 & 4.8 & 8.6 & 12.9 & 17.0 & 19.6 & 19.7 & 16.0 & 12.2 & 8.1 & 5.1 \\
\hline \multirow{3}{*}{ Total fall (mm) } & 2005 & 90.1 & 143.5 & 27.3 & 7.7 & 73.2 & 4.9 & 32.7 & 0.2 & 12.9 & 46.8 & 218.8 & 62.9 \\
\hline & 2006 & 53.2 & 84.7 & 124.0 & 3.8 & 16.7 & 23.0 & 8.2 & 1.2 & 70.6 & 38.0 & 33.9 & 25.6 \\
\hline & Long & 89.7 & 62.4 & 61.9 & 50.6 & 34.4 & 20.8 & 13.3 & 4.2 & 17.1 & 45.8 & 93.6 & 105.8 \\
\hline
\end{tabular}

Long-time is for the period between 1975 and 2006

Table 2. Variance analysis of all analyzed characteristics of the genotypes.

\begin{tabular}{|c|c|c|c|c|c|c|c|c|c|c|}
\hline \multirow[b]{2}{*}{ Source } & \multirow[b]{2}{*}{ DF } & \multicolumn{9}{|c|}{ Mean square } \\
\hline & & BN & PN & PH & FPH & $\mathbf{Y}$ & $1000 \mathrm{SW}$ & C & $\mathrm{C} / \mathrm{N}$ & PO \\
\hline Year $(Y)$ & 1 & 18.34 & $1491.67^{* * *}$ & $132.77^{* * *}$ & $351.48^{* * *}$ & 970254.00 *** & 3462.29 * & 0.00 & 0.00 & 0.02 \\
\hline Planting time (PT) & 1 & $33.75^{* *}$ & $377.40^{* *}$ & $233.95^{* * *}$ & $211.73^{* * *}$ & 89789.29 *** & $10498.95^{* * *}$ & $2.45^{* * *}$ & $104.07^{\star \star *}$ & $309.13^{* * *}$ \\
\hline Genotype (G) & 13 & 7.89 & $202.11^{\star \star *}$ & 39.36 ** & $22.09 * * *$ & $18181.66^{* \star *}$ & $15118.46^{* * *}$ & $0.10^{* * *}$ & $0.85^{* \star \star}$ & $2.69 * \star *$ \\
\hline$G \times Y$ & 13 & 2.16 & 55.32 & 16.99 & 10.56 & 6164.49 * & $5865.03^{\star * *}$ & 0.00 & 0.00 & 0.02 \\
\hline $\mathrm{G} \times \mathrm{PT}$ & 13 & 4.32 & 27.89 & 21.57 & 11.42 & $7965.32^{* * *}$ & 1175.25 & $0.05^{* * *}$ & $0.57^{* \star *}$ & $1.85^{* * *}$ \\
\hline
\end{tabular}

$*, * *$, and ${ }^{* * *}$ : Significant at $0.05,0.01$, and 0.001 , respectively.

Y: Yield, PH: Plant height, FPH: First pod height, BN: Branch number, PN: Pod number, 1000 SW: 1000 seed weight, PO: Protein, C: Carbon.

content in seed were not significantly affected by year. As seen in Table 4, seed yield and seed components for both planting times showed a similar pattern in terms of years.

The fourteen different genotypes grown in both seasons were assessed for several characters and average values of genotypes were presented in Table 5. There were significant differences among the genotypes for all measured characters. In autumn plantings, $99-59 \mathrm{C}\left(2662 \mathrm{~kg} \mathrm{ha}^{-1}\right)$ and $97-102 \mathrm{C}\left(2629 \mathrm{~kg} \mathrm{ha}^{-1}\right)$ lines had the highest seed yield whereas P-3 had the lowest seed yield $\left(744 \mathrm{~kg} \mathrm{ha}^{-1}\right)$. When the genotypes were compared in terms of protein content, P-2 had the highest value $(21.1 \%)$ whereas the lowest value was produced by $97-102 \mathrm{C}(19.6 \%)$ and $97-91 \mathrm{C}$ $(19.7 \%)$. In spring plantings, like in autumn plantings, the highest seed yield was obtained from 99 
Table 3. Effects of planting time on the parameters investigated in the study as two-year average.

\begin{tabular}{|c|c|c|c|c|c|c|c|c|c|}
\hline Planting time & $Y\left(\mathrm{~kg} \mathrm{ha}^{-1}\right)$ & $\mathrm{PH}(\mathrm{cm})$ & BN (number) & PN (number) & FPH (cm) & $1000 \mathrm{SW}(\mathrm{g})$ & PO (\%) & C (\%) & C/N (\%) \\
\hline Autumn & $2050 \mathrm{a}$ & $41.6 \mathrm{a}$ & $6.0 \mathrm{~b}$ & $20.1 \mathrm{~b}$ & $19.0 \mathrm{~b}$ & $435.6 \mathrm{a}$ & $20.5 b$ & $42.48 \mathrm{a}$ & $12.98 \mathrm{a}$ \\
\hline Spring & $1588 b$ & $39.2 \mathrm{~b}$ & $6.9 \mathrm{a}$ & $23.1 \mathrm{a}$ & $21.2 \mathrm{a}$ & $419.7 b$ & $23.2 \mathrm{a}$ & $42.24 b$ & $11.41 b$ \\
\hline $\mathrm{LSD}_{0.05}$ & 179.5 & 1.29 & 0.88 & 2.85 & 0.89 & 8.95 & 0.17 & 0.04 & 0.09 \\
\hline
\end{tabular}

Y: Yield, PH: Plant height, FPH: First pod height, BN: Branch number, PN: Pod number, 1000 SW: 1000 seed weight, PO: Protein, C: Carbon.

Table 4. Effects of planting time on the parameters investigated in the study by years.

\begin{tabular}{|c|c|c|c|c|c|c|c|c|c|c|}
\hline Planting time & Year & $Y\left(\mathrm{~kg} \mathrm{ha}^{-1}\right)$ & $\mathrm{PH}(\mathrm{cm})$ & BN (number) & PN (number) & FPH (cm) & $1000 \mathrm{SW}(\mathrm{g})$ & PO (\%) & C (\%) & $\mathrm{C} / \mathrm{N}(\%)$ \\
\hline & 1. & $3039 \mathrm{a}$ & $40.5 \mathrm{~b}$ & $4.0 \mathrm{~b}$ & $14.2 \mathrm{~b}$ & $21.4 \mathrm{a}$ & $448.5 \mathrm{a}$ & 20.47 & 42.49 & 12.98 \\
\hline Autumn & $\begin{array}{c}2 . \\
\text { LSD }_{0.05}\end{array}$ & $\begin{array}{c}1062 b \\
194.0\end{array}$ & $\begin{array}{c}42.6 \mathrm{a} \\
1.77\end{array}$ & $\begin{array}{l}8.0 \mathrm{a} \\
0.70\end{array}$ & $\begin{array}{c}26.1 \mathrm{a} \\
3.28\end{array}$ & $\begin{array}{c}16.5 b \\
1.14\end{array}$ & $\begin{array}{c}422.6 \mathrm{~b} \\
12.24\end{array}$ & $\begin{array}{c}20.46 \\
0.14\end{array}$ & $\begin{array}{c}42.47 \\
0.05\end{array}$ & $\begin{array}{c}12.98 \\
0.09\end{array}$ \\
\hline & 1. & $2119 a$ & $42.0 \mathrm{a}$ & $8.2 \mathrm{a}$ & 23.1 & 21.7 & 415.9 & 23.19 & 42.24 & 11.40 \\
\hline Spring & $\begin{array}{c}2 . \\
\mathrm{LSD}_{0.05}\end{array}$ & $\begin{array}{c}1057 b \\
207.8\end{array}$ & $\begin{array}{c}36.4 b \\
1.23\end{array}$ & $\begin{array}{l}5.5 b \\
0.98\end{array}$ & $\begin{array}{l}23.1 \\
3.48\end{array}$ & $\begin{array}{l}20.8 \\
1.09\end{array}$ & $\begin{array}{l}423.6 \\
11.85\end{array}$ & $\begin{array}{c}23.16 \\
0.32\end{array}$ & $\begin{array}{c}42.24 \\
0.06\end{array}$ & $\begin{array}{c}11.42 \\
0.16\end{array}$ \\
\hline
\end{tabular}

Y: Yield, PH: Plant height, FPH: First pod height, BN: Branch number, PN: Pod number, 1000 SW: 1000 seed weight, PO: Protein, C: Carbon.

Table 5. Two-year means of the 14 genotypes for various traits based on different planting times.

\begin{tabular}{|c|c|c|c|c|c|c|c|c|c|}
\hline Genotype & $Y\left(k g ~ h a^{-1}\right)$ & $\mathrm{PH}(\mathrm{cm})$ & FPH (cm) & BN (number) & PN (number) & $1000 \mathrm{SW}(\mathrm{g})$ & PO (\%) & $\mathbf{C}(\%)$ & C/N (\%) \\
\hline \multicolumn{10}{|c|}{ Autumn planting time } \\
\hline $99-59 C$ & $2662 \mathrm{a}$ & $42.73 a b c$ & $19.25 \mathrm{bcd}$ & $6.30 \mathrm{a}$ & 22.27 a-d & $402.0 \mathrm{fg}$ & 19.9 de & $42.52 \mathrm{bc}$ & $13.37 \mathrm{ab}$ \\
\hline $97-102 C$ & $2629 \mathrm{a}$ & $41.23 \mathrm{a}-\mathrm{d}$ & $18.50 \mathrm{~b}-\mathrm{f}$ & $6.77 \mathrm{a}$ & $25.13 \mathrm{ab}$ & $445.4 \mathrm{bcd}$ & $19.6 \mathrm{e}$ & $42.34 \mathrm{~d}$ & $13.51 \mathrm{a}$ \\
\hline $97-50 C$ & $2544 a b$ & $41.13 \mathrm{a}-\mathrm{d}$ & $20.33 a-d$ & $5.70 \mathrm{a}$ & $15.97 \mathrm{~cd}$ & 410.2 efg & $20.1 d$ & $42.49 \mathrm{bc}$ & $13.23 \mathrm{~b}$ \\
\hline $97-73 C$ & $2518 a b$ & $41.8 \mathrm{abc}$ & $21.10 \mathrm{abc}$ & $6.00 \mathrm{a}$ & 20.57 a-d & 412.3 efg & $20.8 \mathrm{abc}$ & $42.43 \mathrm{~cd}$ & 12.77 cde \\
\hline $97-91 C$ & $2473 a b$ & $44.60 \mathrm{a}$ & $22.37 \mathrm{a}$ & $6.10 \mathrm{a}$ & $18.27 \mathrm{a}-\mathrm{d}$ & $439.8 \mathrm{~b}-\mathrm{e}$ & 19.7 e & $42.63 \mathrm{a}$ & $13.52 \mathrm{a}$ \\
\hline $97-75 C$ & $2429 a b$ & $43.20 a b c$ & $21.27 a b$ & $5.73 \mathrm{a}$ & $15.93 \mathrm{~cd}$ & $429.5 \mathrm{c}-\mathrm{g}$ & $21.0 \mathrm{ab}$ & $42.40 \mathrm{~cd}$ & $12.60 \mathrm{de}$ \\
\hline $97-101 C$ & $2406 a b$ & $39.87 \mathrm{bcd}$ & $18.23 c-f$ & $5.70 \mathrm{a}$ & 19.27 a-d & $433.0 \mathrm{c}-\mathrm{f}$ & $21.0 \mathrm{ab}$ & $42.41 \mathrm{~cd}$ & $12.60 \mathrm{e}$ \\
\hline$P-1$ & $2237 \mathrm{abc}$ & $43.93 \mathrm{ab}$ & $21.10 \mathrm{abc}$ & $5.37 \mathrm{a}$ & $17.17 \mathrm{bcd}$ & $499.4 \mathrm{a}$ & $20.8 \mathrm{abc}$ & $42.42 \mathrm{~cd}$ & 12.74 cde \\
\hline $97-223 C$ & $2100 \mathrm{bc}$ & $44.53 \mathrm{ab}$ & $18.67 b-e$ & $6.63 \mathrm{a}$ & $26.80 \mathrm{a}$ & $418.8 \mathrm{~d}-\mathrm{g}$ & $20.1 d$ & $42.58 \mathrm{ab}$ & 13.22 b \\
\hline $98-103 C$ & 1827 c & $41.67 a b c$ & $18.20 c-f$ & $5.97 \mathrm{a}$ & $24.50 a b c$ & $399.7 \mathrm{~g}$ & $20.2 d$ & $42.42 \mathrm{~cd}$ & 13.16 b \\
\hline $98-32 C$ & $1826 \mathrm{c}$ & $40.67-d$ & 17.93 def & $5.90 \mathrm{a}$ & 23.07 a-d & $416.1 \mathrm{~d}-\mathrm{g}$ & $20.9 a b c$ & $42.41 \mathrm{~cd}$ & $12.70 \mathrm{cde}$ \\
\hline Cevdet bey & $1199 \mathrm{~d}$ & $36.63 \mathrm{~d}$ & $15.50 \mathrm{f}$ & $5.73 \mathrm{a}$ & $15.43 \mathrm{~d}$ & $466.2 \mathrm{~b}$ & 20.7 bc & $42.59 \mathrm{ab}$ & $12.86 \mathrm{c}$ \\
\hline
\end{tabular}


Table 5. Contd.

\begin{tabular}{|c|c|c|c|c|c|c|c|c|c|}
\hline $\begin{array}{l}P-2 \\
P-3 \\
L_{S D} D_{0.05}\end{array}$ & $\begin{array}{c}1113 d \\
744 d \\
513.36\end{array}$ & $\begin{array}{c}38.64 \mathrm{~cd} \\
41.27-d \\
4.6941\end{array}$ & $\begin{array}{c}15.78 \text { ef } \\
17.47 \text { def } \\
3.0039\end{array}$ & $\begin{array}{c}5.55 \mathrm{a} \\
6.03 \mathrm{a} \\
1.8453\end{array}$ & $\begin{array}{c}18.67 \mathrm{a}-\mathrm{d} \\
18.67 \mathrm{a}-\mathrm{d} \\
8.6839\end{array}$ & $\begin{array}{c}455.4 \mathrm{bc} \\
469.9 \mathrm{ab} \\
32.389\end{array}$ & $\begin{array}{c}21.1 \mathrm{a} \\
20.6 \mathrm{c} \\
0.3668\end{array}$ & $\begin{array}{c}42.66 \mathrm{a} \\
42.42 \mathrm{~cd} \\
0.1215\end{array}$ & $\begin{array}{c}12.65 \mathrm{cde} \\
12.85 \mathrm{~cd} \\
0.2502\end{array}$ \\
\hline \multicolumn{10}{|c|}{ Spring planting time } \\
\hline $99-59 C$ & 2000 a & $42.70 \mathrm{ab}$ & $22.50 \mathrm{ab}$ & $8.77 \mathrm{ab}$ & $29.73 \mathrm{ab}$ & $384.8 \mathrm{c}$ & 22.8 def & $42.09 \mathrm{e}$ & $11.57 \mathrm{a}-\mathrm{d}$ \\
\hline $97-102 C$ & $1864 a b$ & 40.37abc & $21.77 a b c$ & $8.53 \mathrm{abc}$ & $29.13 \mathrm{abc}$ & $406.6 \mathrm{c}$ & $23.3 \mathrm{~cd}$ & $42.16 \mathrm{de}$ & $11.30 \mathrm{de}$ \\
\hline$P-1$ & $1834 a b$ & $39.20 \mathrm{~cd}$ & $20.83 \mathrm{bc}$ & $6.43 \mathrm{bcd}$ & 21.67 b-e & $496.7 \mathrm{a}$ & $23.4 \mathrm{~cd}$ & 42.36abc & $11.31 \mathrm{cde}$ \\
\hline $97-50 C$ & $1661 \mathrm{abc}$ & $40.67 a b c$ & $22.20 \mathrm{ab}$ & $5.67 \mathrm{~d}$ & $19.10 \mathrm{de}$ & $393.6 \mathrm{c}$ & $23.3 \mathrm{~cd}$ & $42.28 \mathrm{bcd}$ & $11.36 \mathrm{cde}$ \\
\hline$P-2$ & $1651 \mathrm{abc}$ & $37.60 \mathrm{cde}$ & 21.73abc & $5.30 \mathrm{~d}$ & $16.47 \mathrm{e}$ & $474.8 \mathrm{ab}$ & 22.6 def & $42.44 \mathrm{a}$ & $11.72 \mathrm{abc}$ \\
\hline $97-91 C$ & $1628 \mathrm{abc}$ & $42.97 \mathrm{a}$ & $23.80 \mathrm{a}$ & 7.70 a-d & 25.43 a-e & $408.6 \mathrm{c}$ & $22.2 \mathrm{f}$ & $42.20 \mathrm{de}$ & $11.91 \mathrm{a}$ \\
\hline $97-73 C$ & $1602 \mathrm{abc}$ & $40.20 a b c$ & $21.87 a b c$ & $7.60 \mathrm{a}-\mathrm{d}$ & 23.47 a-e & $385.6 \mathrm{c}$ & $24.6 \mathrm{a}$ & $42.07 \mathrm{e}$ & $10.71 \mathrm{f}$ \\
\hline $97-223 C$ & $1567 \mathrm{abc}$ & $37.63 \mathrm{cde}$ & $19.17 \mathrm{c}$ & $6.67 \mathrm{bcd}$ & $28.13 \mathrm{a}-\mathrm{d}$ & $396.3 \mathrm{c}$ & 23.0 cde & $42.18 \mathrm{de}$ & $11.47 \mathrm{~b}-\mathrm{e}$ \\
\hline $97-101 C$ & $1548 a b c$ & $36.90 \mathrm{de}$ & $20.80 \mathrm{bc}$ & $6.10 \mathrm{~cd}$ & 20.33 cde & $407.9 \mathrm{c}$ & 23.0 cde & $42.20 \mathrm{de}$ & $11.45 b-e$ \\
\hline $97-75 C$ & $1508 \mathrm{abc}$ & 34.77 e & 20.27 bc & $5.60 \mathrm{~d}$ & $18.73 \mathrm{e}$ & $392.2 \mathrm{c}$ & $23.0 \mathrm{c}-\mathrm{f}$ & 42.26bcd & $11.52 \mathrm{a}-\mathrm{d}$ \\
\hline $98-32 C$ & 1445 bc & $39.50 \mathrm{bcd}$ & $20.53 \mathrm{bc}$ & $5.67 \mathrm{~d}$ & 21.27 b-e & $388.7 \mathrm{c}$ & $24.4 \mathrm{ab}$ & $42.25 \mathrm{~cd}$ & $10.84 \mathrm{f}$ \\
\hline$P-3$ & 1377 bc & 37.97cde & 20.17 bc & $6.07 \mathrm{~cd}$ & 20.43 cde & $497.3 \mathrm{a}$ & 22.8 def & 42.36abc & 11.64 a-d \\
\hline $98-103 C$ & 1355 bc & $40.77 a b c$ & $20.53 b c$ & $9.70 \mathrm{a}$ & $32.13 \mathrm{a}$ & $379.6 \mathrm{c}$ & 23.8 bc & $42.09 \mathrm{e}$ & 11.10 ef \\
\hline Cevdet bey & 1194 c & 37.67cde & 20.97abc & $6.23 \mathrm{bcd}$ & $17.63 \mathrm{e}$ & $463.6 \mathrm{~b}$ & 22.4 ef & $42.41 \mathrm{ab}$ & $11.86 a b$ \\
\hline $\mathrm{LSD}_{0.05}$ & 549.73 & 3.2485 & 2.8855 & 2.5853 & 9.1961 & 31.36 & 0.8354 & 0.1535 & 0.412 \\
\hline
\end{tabular}

Y: Yield, PH: Plant height, FPH: First pod height, BN: Branch number, PN: Pod number, 1000 SW: 1000 seed weight, PO: Protein, C: Carbon. Genotypes designated with different letters are different from each other at $5 \%$ significance level.

- 59C $\left(2000 \mathrm{~kg} \mathrm{ha}^{-1}\right)$. The lowest yield was obtained from Cevdet Bey (1194 kg ha-1) which was the standard variety. The highest protein content was found in $97-73 \mathrm{C}$ with $24.6 \%$ while the lowest protein content was found in $97-91 \mathrm{C}$ with $22.2 \%$. When seed yield are considered for both years, line 99 - 59C for autumn planting was a promising genotype with its high seed yield, even its lower protein content and 1000-seed weight.

Simple correlation coefficients among the traits were given in Table 6 . The yield showed a significant positive relationships with $\mathrm{PH}\left(r=0.21^{* * *}\right)$, FPH $\left(r=0.52^{\star \star \star}\right)$, PN $\left(r=0.40^{\star \star *}\right)$, and $\mathrm{C} / \mathrm{N}(r=$ $\left.0.20^{\star *}\right)$, whereas a significant negative correla- tions with $B N\left(r=-0.29^{* \star *}\right)$ and $P O\left(r=-0.19^{* *}\right)$. However, correlation coefficients were very low, except for FPH and PN. This means that seed yield increased with increasing number of pods (PN). And also PO was positively correlated with FPH $\left(r=0.29^{\star \star \star}\right)$. Correlation analysis showed that $\mathrm{PO}$ was significantly and negatively correlated with $\mathrm{PH}\left(r=-0.21^{\star * *}\right), 1000 \mathrm{SW}\left(r=-0.19^{\star *}\right)$, $C\left(r=-0.61^{* \star \star}\right)$ and $C / N\left(r=-0.99^{\star \star \star}\right)$. Similar to our results, various researchers stated that there was a positive correlation between yield and $\mathrm{PH}$, PN and FPH (Uddin et al., 1990; Güler et al., 2001; Yücel et al., 2006). Katiyar et al. (1981) conducted a study to determine the relationships among the traits affecting yield in chickpea and found that number of pods per plant had the highest direct effect on seed yield. Yücel et al. (2006) reported that FPH and number of seeds per plant were good selection criteria in kabuli winter chickpea for yield improvement.

In conclusion, this two-year experiment demonstrated that autumn planting with cold and disease resistant chickpea genotypes significantly increased the seed yield. Similarly planting times also significantly affected seed protein content, $\mathrm{C}$ and $\mathrm{C} / \mathrm{N}$ ratio. Thus it can be said that genotype and environmental conditions are the main factors influencing the seed yield and protein content in 
Table 6. Correlation coefficients among the characteristics of the genotypes investigated.

\begin{tabular}{|c|c|c|c|c|c|c|c|c|}
\hline Character & BN & PN & PH & FPH & $\mathbf{Y}$ & $1000 \mathrm{SW}$ & C & $\mathrm{C} / \mathrm{N}$ \\
\hline PN & $0.81^{* \star *}$ & - & & & & & & \\
\hline $\mathrm{PH}$ & $0.35^{* * *}$ & $0.26^{* \star *}$ & - & & & & & \\
\hline FPH & $-0.28^{* * *}$ & $-0.38^{\star * *}$ & $0.29^{* \star *}$ & - & & & & \\
\hline Y & $-0.29^{* * *}$ & $0.40^{* * *}$ & $0.21^{* \star *}$ & $0.52^{\star \star *}$ & - & & & \\
\hline 1000 SW & $-0.32^{\star * *}$ & $-0.29^{\star \star *}$ & -0.09 & 0.04 & 0.06 & - & & \\
\hline C & $-0.23^{\star \star *}$ & $-0.23^{\star \star *}$ & 0.07 & $-0.22^{\star \star \star}$ & 0.06 & $0.34^{* * *}$ & - & \\
\hline $\mathrm{C} / \mathrm{N}$ & -0.12 & -0.09 & $0.22^{* * *}$ & $-0.29^{\star * *}$ & $0.20^{* *}$ & $0.19^{\star *}$ & $0.65^{\star \star \star}$ & - \\
\hline $\mathrm{PO}$ & -0.11 & 0.07 & $-0.21^{* * *}$ & $0.29^{\star \star *}$ & $-0.19^{* *}$ & $-0.19^{\star *}$ & $-0.61^{* * *}$ & $-0.99^{\star * *}$ \\
\hline
\end{tabular}

${ }^{* *}$ and ${ }^{* * *}$ : Significant at 0.01 and 0.001 , respectively.

chickpea. During cold winter conditions, when the field surface is covered by snow, the plant survival is relatively higher than it is with no-snow conditions. Freezing (below $\left.-1.5^{\circ} \mathrm{C}\right)$ and chilling $\left(-1.5\right.$ to $\left.+15^{\circ} \mathrm{C}\right)$ temperatures affect chickpea at different development stages (Croser et al., 2003). However, some genotypes (P-1, P-2, P-3 and standard variety Cevdet Bey) were severely affected by cold and disease. Therefore, for superior yield and quality, especially cold resistant and/or tolerant genotypes should be bred in sub-tropic areas and Mediterranean countries. This study provides clear evidences for successful adaptation of new chickpea genotypes grown in autumn conditions in northwest Turkey, and demonstrates important changes in yield, yield components and chemical properties due to changes in planting time. The production of chickpea with autumn planting will be less affected by irregular precipitation during growing season, providing higher and more stable yields over the years.

\section{ACKNOWLEDGMENTS}

This research was financially supported by TUBITAK (TOVAG-105-O-346) and Çanakkale Onsekiz Mart University (BAP-2005/94).

\section{REFERENCES}

Akçin A (1988). Yemeklik Dane Baklagiller. Selçuk Üniversitesi Yayınları 43 Ziraat Fakültesi Yayınları 8: 377.

Almeida Costa GE, Silva Queiroz-Monici K, Machado Reis SMP, Oliveira AC (2006). Chemical composition, dietary fibre and resistant starch contents of raw and cooked pea, common bean, chickpea and lentil legumes. Food Chem. 94: 327-330.

Amjad I, Khalil IA, Shah H (2003). Nutritional yield and amino acid profile of rice protein as influenced by nitrogen fertilizer. Sarhad J. Agric. 19: 127-134.

Berger JD, Ali M, Basu PS, Chaudhary BD, Chaturvedi SK, Deshmukh PS, Dharmaraj PS, Dwivedi SK, Gangadhar GC, Gaur PM, Kumar J, Pannu RK, Siddique KHM, Singh DN, Singh DP, Singh SJ, Turner NC, Yadava HS, Yadav SS (2006). Genotype by environment studies demonstrate the critical role of phenology in adaptation of chickpea (Cicer arietinum L.) to high and low yielding environments of India. Field Crops Res. 98: 230-244.

Calcagno F, Gallo G, Raimondo J, Venora G, Jatani M (1987). Early planting to increase and stabilize chickpea crop in hot dry environ- ments. ENED La Coltura del Cece in Italia, 130-131.

Croser JS, Clarke HJ, Siddique KHM, Khan TN (2003). Low temperature stress: implications for chickpea (Cicer arietinum L.) improvement. Crit. Rev. Plant Sci. 22: 185-219.

El-Adawy TA (2002). Nutritional composition and antinutritional factors of chickpeas (Cicer arietinum L.) undergoing different cooking methods and germination. Plant Foods Hum. Nutr. 57: 83-97.

El Hardallon SB, Salih FA (1981). Chemical characteristics of twenty four cultivars of kabuli type of chickpea grown in Sudan. Legume Res. 4: 14-18.

Farzana W, Khalil IA (1999). Protein quality of tropical food legumes. J. Sci. Technol. 23: 13-19.

Fernando Flores AS, Hernandez M (1987). Chemical composition and nutritional value of Mexican varieties of chickpea (Cicer arietinum L.). Plant Foods Hum. Nutr. 37: 299-306.

Ghirardi P, Marzo A, Ferrari G (1974). Lipid classes and total fatty acids pattern of Cicer arietinum. Phytochemistry. 13: 755-756.

Gül MK, Egesel CÖ, Türk FM (2005). Bazı nohut hatlarının Çanakkale yöresinde kış koşullarına adaptasyonu. Türkiye VI. Tarla Bitkileri Kongresi, 5-9 Eylül 2005, Antalya, Araştırma Sunusu Cilt I: 267-269.

Gül MK, Egesel CÖ, Turhan H (2008). The effects of planting time on fatty acids and tocopherols in chickpea. Eur. Food Res. Technol. 226(1): 517-522.

Güler M, Adak MS, Ulukan H (2001). Determining relationships among yield and some yield components using path coefficient analysis in chickpea (Cicer arietinum L.). Eur. J. Agron. 14: 161-166.

Hawtin GC, Singh KB (1984). Prospects and potential of winter sowing of chickpea in the Mediterranean region. In: eds. Saxena MC Singh $\mathrm{KB}$, Ascochyta Blight and Winter Sowing of Chickpea. Martinus Nijhoff/Dr. W. Junk Publishers, The Hague, The Netherlands, pp 716.

Hulse JH (1991). Nature, composition and utilization of grain legumes. In: Uses of tropical Legumes: Proceedings of a Consultants' Meeting. 27-30 March 1989, ICRISAT Center ICRISAT, Patancheru, A.P. 502 324 India. pp 11-27.

lliadis C (2001). Evaluation of six chickpea varieties for seed yield under autumn and spring sowing. J. Agric. Sci. 137: 439-444.

Iqbal A, Khalil IA, Ateeq N, Khan MS (2006). Nutritional quality of important food legumes. Food Chem. 97: 331-335.

Katiyar RP, Sood OP, Kalia NR (1981). Selection criteria in chickpea. International Chickpea Newslett. 4: 5-6.

Kün E (1988). Serin İklim Tahılları. Ankara Üniversitesi Ziraat Fakültesi Yayınları 1032, Ders Kitabı 299: 322.

Monti LM, Grillo S (1983). Legume seed improvement for protein content and quality. Qual. Plant Plant Foods Hum. Nutr. 32: 253-266.

Özdemir S, Karadavut U (2003). Comparison of the performance of autumn and spring sowing of chickpeas in a temperate region. Turk. J. Agric. Fore. 27: 345-352.

SAS Institute Inc (1999) SAS/STAT Version 8. Cary, NC

Singh KB (1997). Chickpea (Cicer arietinum L.). Field Crops Res. 53: 161-170.

Singh KB, Malhotra RS, Saxena MC, Bejiga G (1997). Superiority of winter sowing over traditional spring sowing of chickpea in the Medi- 
terranean region. Agron. J. 89: 112-118.

Tayyar Ş, Gül MK, Egesel CÖ (2005). Bazı nohut (Cicer arietinum L.) hatlarının kışa mukavemetlerinin belirlenmesi. Türkiye VI. Tarla Bitkileri Kongresi, 5-9 Eylül 2005, Antalya, Araştırma Sunusu Cilt I: 271-273.

Uddin MJ, Hamid MA, Rahman ARMS, Newaz MA (1990). Variability, correlation and path analysis in chickpea (Cicer arietinum L.) in Bangladesh. Bangladesh J. Plant Breed. Genet. 3: 51-55.
Yücel DÖ, Anlarsal AE, Yücel C (2006). Genetic variability, correlation and path analysis of yield, and yield components in chickpea (Cicer arietinum L.). Turk. J. Agric. Forest. 30: 183-188. 